\title{
Exploration of acetylation as a base-labile protecting group in Escherichia coli for an indigo precursor
}

Luke N. Latimer ${ }^{1}$, Zachary N. Russ ${ }^{2,3}$, James Lucas ${ }^{2,3}$, John E. Dueber ${ }^{3 *}$

1 Department of Chemistry, University of California, Berkeley, Berkeley, California, 94720, USA

2 The UC Berkeley \& UCSF Graduate Program in Bioengineering, Berkeley, California, 94720, USA

3 Department of Bioengineering, University of California, Berkeley, Berkeley, California, 94720, USA

*To whom correspondence should be addressed. Tel: +1 510643 4616; Fax: +1 510642 9725;

University of California, Berkeley, 2151 Berkeley Way, Room 512D, Berkeley CA, 94704

Email: jdueber@berkeley.edu

\section{SUPPORTING INFORMATION}

Supporting Table S1

Supporting Table S2

Supporting Figure S1

Supporting Figure S2

Supporting Figure S3

Supporting Figure S4

Supporting Methods

Supporting References
DNA oligomer sequences

Plasmid descriptions

Indirubin extractions from dyed swatches

Hydrolase knockout phenotypic analysis

Ester stability

Indoxyl acetate toxicity 
Supporting Table S1. DNA oligomer names and sequences. Underlined sequences indicate restriction recognition sites.

\begin{tabular}{|c|c|}
\hline Oligo Name & Sequence \\
\hline aes for & CCGGCGCTTAATCCATAACG \\
\hline aes seq-r & GGTGTAATCAATACCAATCACCG \\
\hline aes MASC WT-r & AGTTTGTTTTCCGGCTTCAT \\
\hline aes MASC KO-r & AGTTTGTTTTCCGGCTTCAC \\
\hline bioH for & AGACGAAAGGCATTTTTCAGG \\
\hline bioH MASC WT-r & TCTGCCACCAGATGTTATTcat \\
\hline bioH MASC KO-r & TGCCACCAGATGTTATTcacta \\
\hline yjfP seq-f & CAGGCACTTAACATCAACACC \\
\hline yjfP rev & CCAGAATTGATTTAACCGCCG \\
\hline yjfP MASC WT-f & CCAGACAAAAAGGAGAGACAGA \\
\hline yjfP MASC KO-f & CCAGACAAAAAGGAGAGACAtAg \\
\hline yeiG seq-f & GCTTCTTTACTGAGTGATGGC \\
\hline yeiG rev & GCGTGGCATTAAGATAAAAGCC \\
\hline yeiG MASC WT-f & CAACAAGGAGCCACGCA \\
\hline yeiG MASC KO-f & ACAACAAGGAGCCACGTAG \\
\hline frmB for & GGGCATGGTTGAAGATGCG \\
\hline frmB seq-r & CAATAATGTTGTGCTCAGCCG \\
\hline frmB MASC WT-r & ACATGTTTTTCAATGAGTTCCATC \\
\hline frmB MASC KO-r & CATGTTTTTCAATGAGTTCCACTA \\
\hline entH seq-f & CGCCAACACGATTTTGTTCC \\
\hline entH rev & CACACTGTCCGTCGCGGGTCA \\
\hline entH MASC WT-f & CTGGGGGCATAAGCatgATCTGGAAACG \\
\hline entH MASC KO-f & CGCTGGGGGCATAAGCatgATCTaGtAAtGa \\
\hline
\end{tabular}




\begin{tabular}{|c|c|}
\hline ydil seq-f & GCCATTACACGatgAACGC \\
\hline ydiL rev & ACCCGCGGCATTAGTCTGCCA \\
\hline ydiL MASC WT-f & GACAGTGAAAGCTGGCGTCAGTGGG \\
\hline ydiL MASC KO-f & GGTGACAGTGAAAGCTGGCGTtAGTGat \\
\hline tes $A$ seq- $f$ & CCAGCAAGGTTGACTTACC \\
\hline tesA rev & CCAGCACATGAAAACCCTGGCG \\
\hline tesA MASC WT-f & AAGatgATGAACTTCAACAATGTTTTCCGCTGGC \\
\hline tesA MASC KO-f & GACTTCTTAAGatgATGAACTTCAACAATGTTTTCCGCTGatAaTa \\
\hline nanS seq-f & ACAGTACGGCACCGTTGGCC \\
\hline nans rev & TCTTTCCAGTACCAGGTGGTATCGCC \\
\hline nanS MASC WT-f & CGGGCAGCGAAGGGACATATTCAG \\
\hline nanS MASC KO-f & CGGGCAGCGAAGGGACATAgTgAt \\
\hline yqiA seq-f & GGCGGACACACGTTTCCAACC \\
\hline yqiA rev & CGGTGAGTACCTCAATGGCATCAGCG \\
\hline yqiA MASC WT-f & GCGCTCTGCAAAAGCGAGCTTGTTAA \\
\hline yqiA MASC KO-f & GCGCTCTGCAAAAGCGAGCTaGTgAt \\
\hline ybfF seq-f & CCCTAACCCGACAACGCG \\
\hline ybfF rev & CGATCGCCACCAGTTTATCGATGC \\
\hline ybfF MASC WT-f & GTTGATATGCGTAACCACGGTCTTTCACCGA \\
\hline ybfF MASC KO-f & CCAGGTTGATATGCGTAACCACGGTCTTTaAtaGt \\
\hline ypfH seq-f & GTTTGCACCGCTGTTTCC \\
\hline ypfH rev & GGTATAACGCAAGTGATCCAGG \\
\hline ypfH MASC WT-f & CGGTGCGCTACTGGCA \\
\hline ypfH MASC KO-f & TGAAACGGTGCGCTAaTGat \\
\hline frs $A$ seq-f & CATGATTAACCGTCTGATGTGG \\
\hline frsA rev & AACGTTAGCGCCGAAACG \\
\hline
\end{tabular}




\begin{tabular}{|c|c|}
\hline frsA MASC WT-f & GGCAACTGGATTTATGAGTGGG \\
\hline frsA MASC KO-f & GCGGCAACTGGATTTAatAGTGa \\
\hline tnaA seq- $f$ & TTTTAGTAAATGATGGTGCTTGCATA \\
\hline tnaA rev & TGGTGATGGTTGCAACG \\
\hline tnaA MASC WT-f & CACTCGCGCTTATCGTG \\
\hline tnaA MASC KO-f & CCACTCGCGCTTAgtGat \\
\hline J23110 RBS lib-r & ttaaGGTCTCtcatagaHHHHYYYYYHHNNNNgaagctagcgctagcattgtac \\
\hline ScATF1-f1 & GCATCGTCTCATCGGTCTCATATGAATGAAATCGATGAGAAAAATCAGG \\
\hline ScATF1-r1 & ATGCCGTCTCAATTCаTCTCTTAAATCATGAAAAAAGTGGATC \\
\hline ScATF1-f2 & GCATCGTCTCAGAATTAAATAATATTAAAACTCCACCAAAAAAATTA \\
\hline ScATF1-r2 & ATGCCGTCTCAGGTCTCAGGATCCAGGGCCTAAAAGGAGAGC \\
\hline ScATF2-f & GCATCGTCTCATCGGTCTCATATGgaagatatagaaggatacgaacc \\
\hline ScATF2-r & ATGCCGTCTCAGGTCTCAGGATCCaagcgacgcaaattcgcC \\
\hline ScEEB1-f & GCATCGTCTCATCGGTCTCATTCTtttcgctcgggttactatc \\
\hline ScEEB1-r & ATGCCGTCTCAGGTCTCAGGATCCtaaaactaactcatcaaagctgc \\
\hline ScEHT1-f & GCATCGTCTCATCGGTCTCCTATGtcagaagtttccaaatggCC \\
\hline ScEHT1-r & ATGCCGTCTCAGGTCTCAGGATCCtacgactaattcatcaaacttagtg \\
\hline AbWS/DGAT-f & TATCGGTCTCATATGtcttataagaataaccattctattttatc \\
\hline CAT-f & GCATCGTCTCATCGGTCTCATATGgagaaaaaatcactggatatacC \\
\hline CAT-r & ATGCCGTCTCAGGTCTCAGGATCCcgccccgccetgc \\
\hline CbBEAT1-f & TATCGGTCTCATATGaatgttacaatgcattcaaag \\
\hline FaAAT2-f & TATCGGTCTCATATGtcttataagaataaccattctattttatc \\
\hline FaSAAT-f & TATCGGTCTCATATGgagaaaatcgaggtttcg \\
\hline PhCFAT-f & TATCGGTCTCATATGggtaacaccgacttcc \\
\hline RhAAT1-f & TATCGGTCTCATATGgagaagatcgaagtctc \\
\hline RsVS-f & TATCGGTCTCATATGgcaccgcagatgg \\
\hline
\end{tabular}


Oligomers used for knockout recombineering

\begin{tabular}{|c|c|}
\hline aes KO & $\begin{array}{l}\text { T*G*GCAGTGGTTCCTTACAATGACGTAATTTGAAAGGAGTTTTTGTAGTGAAG } \\
\text { CCGGAAAACAAACTACCTGTTCTGGACCTTATTTCTGC }\end{array}$ \\
\hline bioH KO & $\begin{array}{l}\text { T*T*GGTCTGCCACCAGATGTTATTCACTATCCGCTATTGTTCTCTTTTGACTT } \\
\text { ACAAGGATGAACATATGCTAACAGTACCGGGATTATGC }\end{array}$ \\
\hline yjfP KO & $\begin{array}{l}\text { A*A*GAACGGGAATATCTGCCAGCTCGCGTGATTCTATTTCAATCACTATGTCT } \\
\text { CTCCTTTTTGTCTGGTATGACATGAAGAAATCCTTAGC }\end{array}$ \\
\hline yeiG KO & $\begin{array}{l}\text { A*A*ACGGTATGATGAAGAAATTGCAAACAACACAACAAGGAGCCACGTAGTGG } \\
\text { AAATGCTCGAAGAGCACCGCTGTTTTGAAGGCTGGCAG }\end{array}$ \\
\hline frmB KO & $\begin{array}{l}\text { T*A*ATACTCTCCCCGGGCAGCCGTCCGGGGGATTAACCCTGAGATAATGACTT } \\
\text { AGTGGAACTCATTGAAAAACATGTCAGCTTTGGCGGCT }\end{array}$ \\
\hline entH KO & $\begin{array}{l}\text { G*C*GTTCAGTTCGTCGAGCGTTAAATGtCaTTaCtAGATCATGCTTATGCCCC } \\
\text { CAGCGTTGAGCCGCCATCGACCACAATATCCTGTAGGG }\end{array}$ \\
\hline ydiL KO & $\begin{array}{l}\text { G*A*TTGCCCAGACCGGTGACAGTGAAAGCTGGCGTtAGTGatAAAATGGCAAG } \\
\text { TGTGCCATTCCTGATCGTGTAGTCGAGCAACTGTTGGC }\end{array}$ \\
\hline tesA KO & $\begin{array}{l}\text { G*A*CTTCTTAAGATGATGAACTTCAACAATGTTTTCCGCTGatAaTaGCCCTT } \\
\text { CCTGTTCCTGGTCCTGTTAACCTTCCGTGCCGCCGCAG }\end{array}$ \\
\hline nanS KO & $\begin{array}{l}\text { G*C*GGATCGGCTTTTACCGCGGGCAGCGAAGGGACATAgTgAtAACGGCACGG } \\
\text { AGCCAGCCATGATGCTTGTCGTTGGGGAACGGATACTC }\end{array}$ \\
\hline yqiA KO & $\begin{array}{l}\text { C*C*GCCAGCCAGTTTTaTCACtAGCTCGCTTTTGCAGAGCGCGGCGAGCTGTT } \\
\text { GAAACCGTGTAAATAAAGAAGCGTAGACATCAGTAGCC }\end{array}$ \\
\hline ybfF KO & $\begin{array}{l}\text { C*A*GGTTGATATGCGTAACCACGGTCTTTaAtaGtGAGATCCGGTAATGAATT } \\
\text { ACCCGGCGATGGCGCAGGATCTTGTTGATACTCTGGAT }\end{array}$ \\
\hline ypfH KO & $\begin{array}{l}\text { G*C*CCCTACTCCGCTCTGTTTCTatCAtTAGCGCACCGTTTCAATAAACGTCG } \\
\text { GCATGATCGCATCCACACGCGCCTGGCGATTATCTTCC }\end{array}$ \\
\hline frsA KO & $\begin{array}{l}\text { C*A*CACCATCGCCTGGGTGGCtCACTatTAAATCCAGTTGCCGCCACGGTAGC } \\
\text { CAATCACCGTATCGTATAAATCATCGTCGGTACGTTCG }\end{array}$ \\
\hline $\operatorname{tnaA} \mathrm{KO}$ & $\begin{array}{l}\text { G*A*ACCGTTCCGCATTCGTGTTATTGAGCCAGTAAAACGTACCACTCGCGCTT } \\
\text { AgtGatAAGAGGCAATTATTAAATCCGGTATGAACCCG }\end{array}$ \\
\hline
\end{tabular}

* indicates phosphorothioate bonds. 
Supporting Table S2. Plasmids used in this study. Plasmids sequences are available on addgene (https://www.addgene.org/).

\begin{tabular}{|c|c|c|}
\hline Plasmid & Description & Figure \\
\hline pLNL1126 & 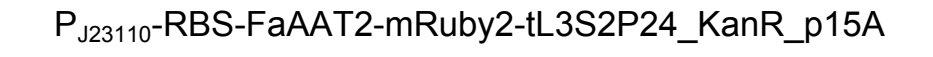 & $3 \mathrm{~A}$ \\
\hline pLNL1127 & $P_{\text {J23110-RBS-RhAAT1-mRuby2-tL3S2P24_KanR_p15A }}$ & $3 \mathrm{~A}$ \\
\hline pLNL1128 & $P_{\text {J23110-RBS-CbBEAT-mRuby2-tL3S2P24_KanR_p15A }}$ & $3 A$ \\
\hline pLNL1130 & 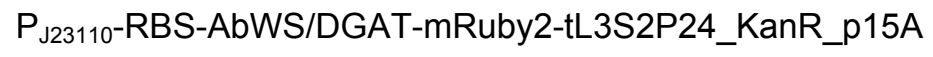 & $3 \mathrm{~A}$ \\
\hline pLNL1131 & 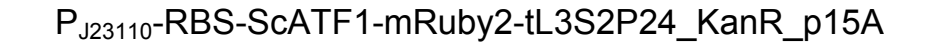 & $3 \mathrm{~A}$ \\
\hline pLNL1132 & 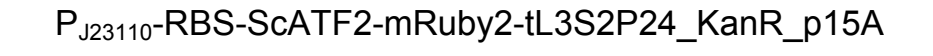 & $3 \mathrm{~A}$ \\
\hline pLNL1133 & 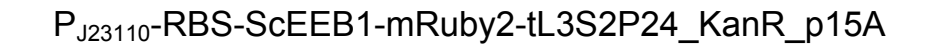 & $3 \mathrm{~A}$ \\
\hline pLNL1134 & 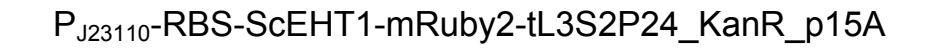 & $3 \mathrm{~A}$ \\
\hline pLNL1135 & $P_{\text {J23110-RBS-CAT-taa-mRuby2-tL3S2P24_KanR_p15A }}$ & $3 \mathrm{~A}$ \\
\hline pLNL1141 & 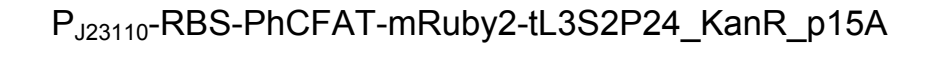 & $3 \mathrm{~A}$ \\
\hline pLNL1142 & $P_{\text {J23110-RBS-FvSAAT-mRuby2-tL3S2P24_KanR_p15A }}$ & $3 \mathrm{~A}$ \\
\hline pLNL1143 & 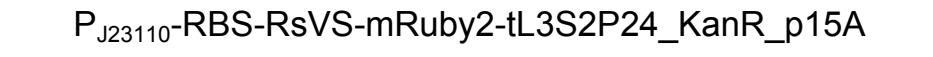 & $3 \mathrm{~A}$ \\
\hline pLNL1151 & 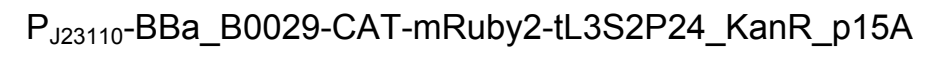 & 3B \\
\hline
\end{tabular}


A

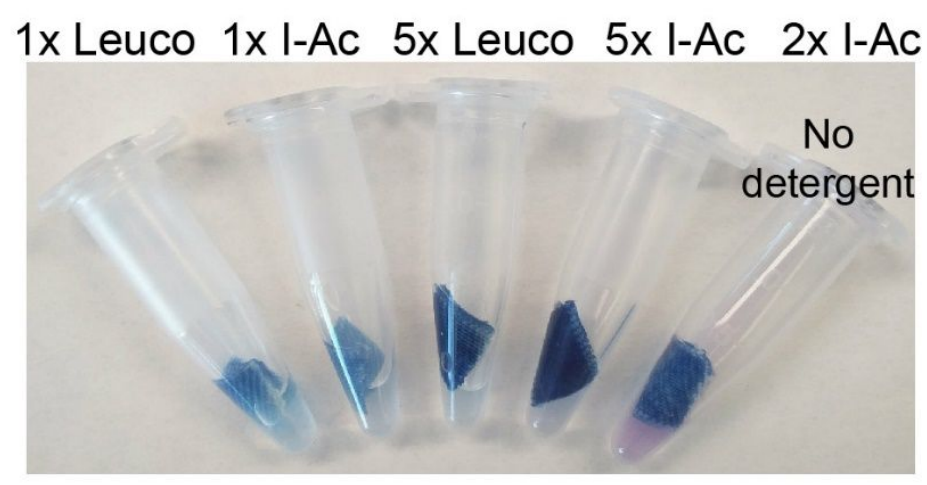

B

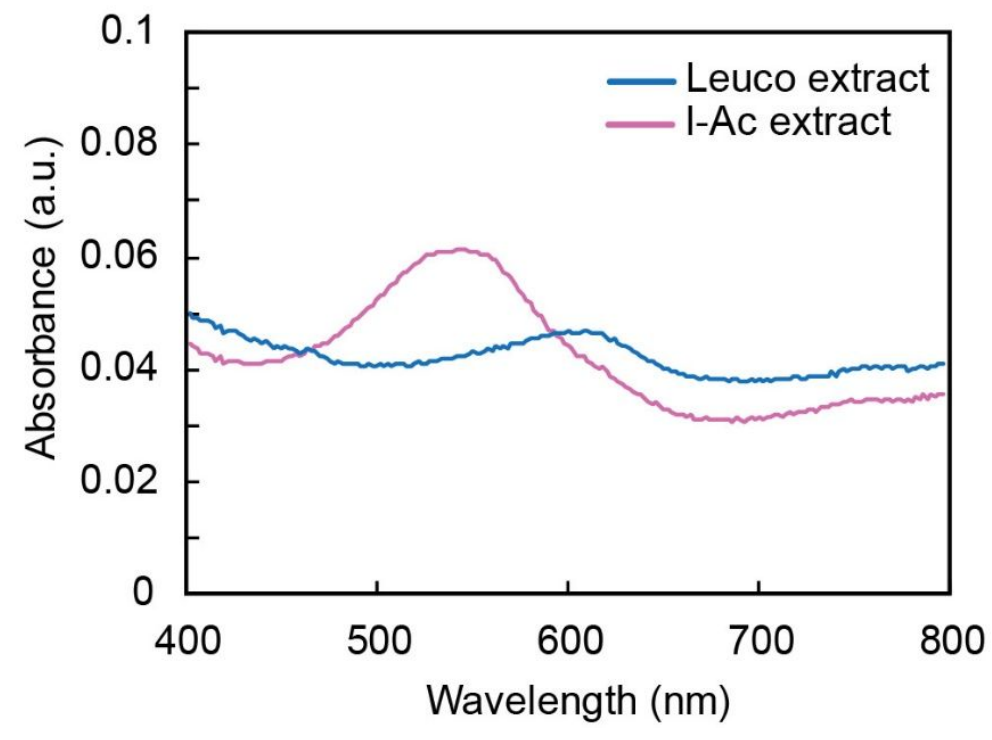

Supporting Figure S1. Methanol extraction of dyed swatches.

(A) $5 \mathrm{~mm}^{2}$ sections of swatches dyed using leucoindigo prepared from indigo ("leuco") or dyed with indoxyl acetate ("I-Ac"). All swatches were laundered with detergent (Kirkland Signature Ultra Clean Liquid Laundry Detergent) in a Whirlpool washing machine with "Normal" settings before extract except the one of the right labeled "no detergent" which was washed in DI water. For extraction, swatches were vortexed with $100 \mu \mathrm{L}$ of methanol for $1 \mathrm{~min}$. The number indicates the number of dye applications. (B) Absorbance scan using a TECAN M1000 of the methanol extractions from (A). 
A

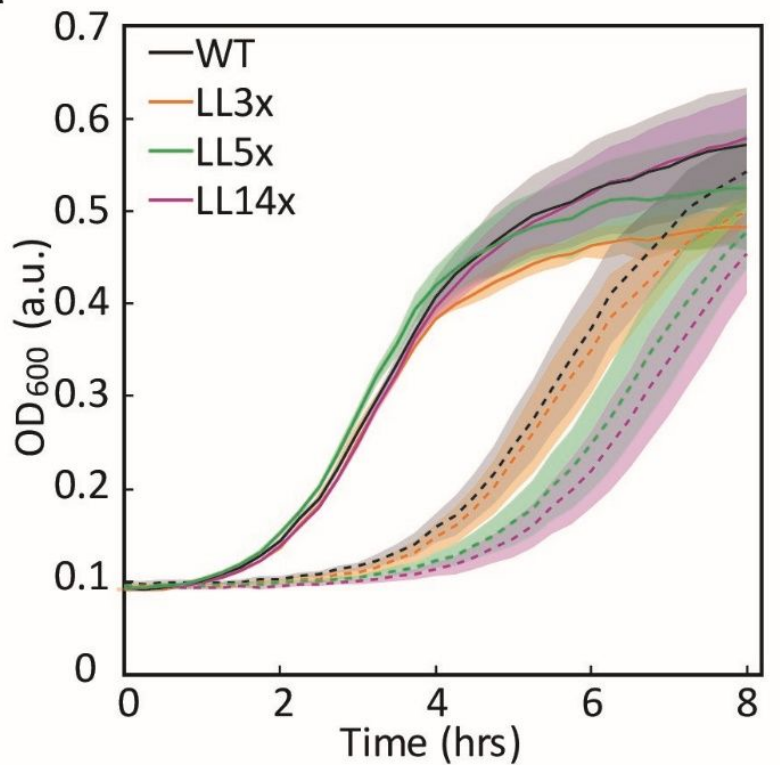

B

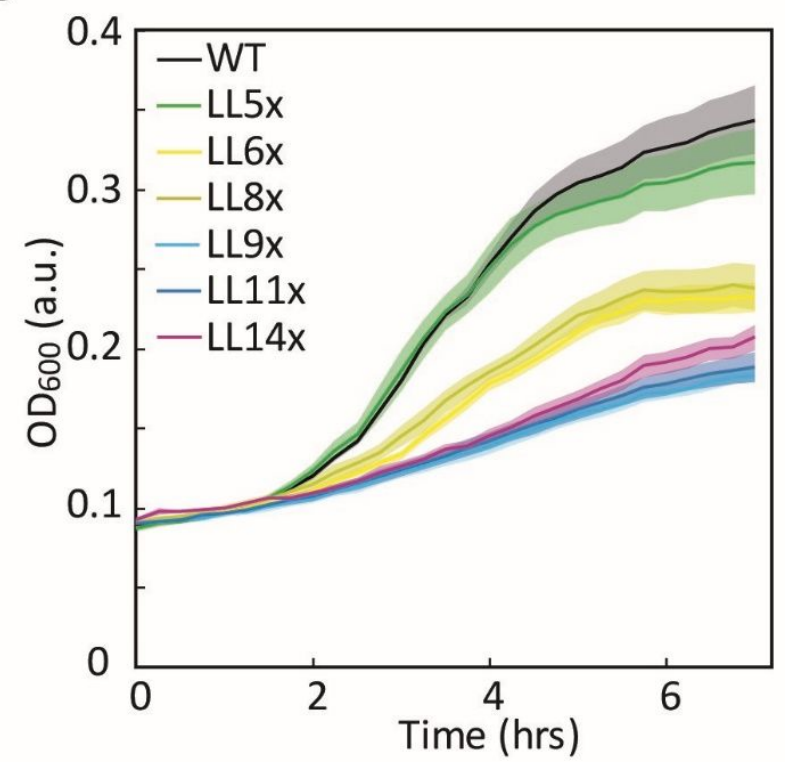

Supporting Figure S2. Hydrolase knockout phenotypic analysis shows expected sensitivities.

A) Formaldehyde sensitivity. Various knockout strains were grown to saturation and back diluted 1:220 in LB with (dashed) or without (solid) $800 \mu \mathrm{M}$ formaldehyde and grown in microtiter plates. Double knockout of frmB and yeiG (LL5x) are reported to have a growth defect in formaldehyde ${ }^{1}$.

B) Salicylate sensitivity. Various knockout strains were grown to saturation and diluted 1:200 in LB with $250 \mu \mathrm{M}$ dipyridyl and $350 \mu \mathrm{M}$ salicylate in microtiter plates. Knockout of entH (LL6x) is reported to have a growth defect in these conditions ${ }^{2}$. Shaded areas represent standard deviation of six biological replicates. 
A

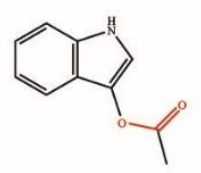

Indoxyl acetate

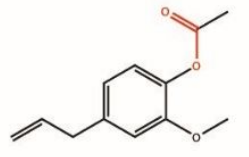

Eugenol acetate

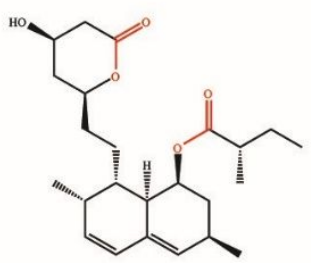

Lovastatin

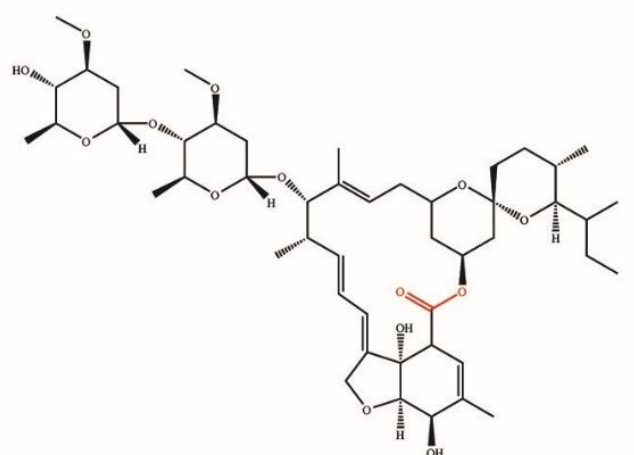

Ivermectin

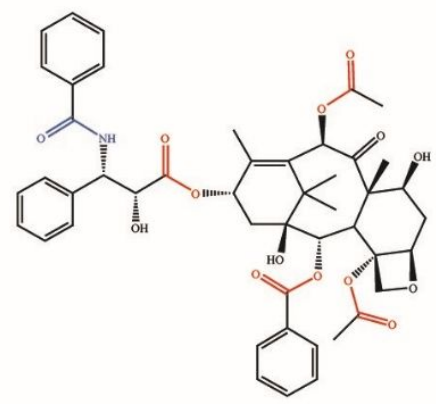

Paclitaxel

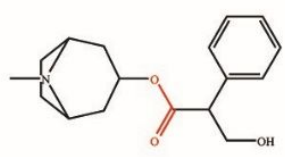

Atropine

B

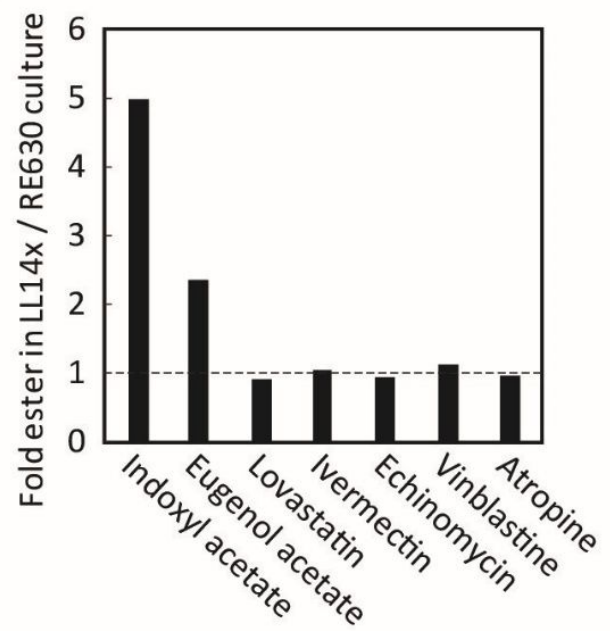

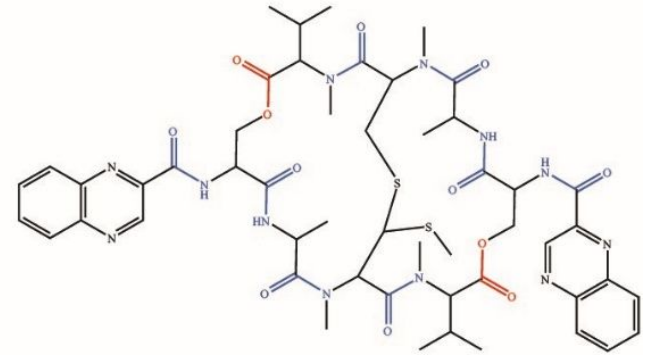

Echinomycin

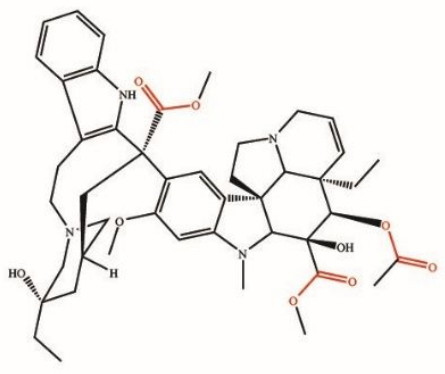

Vinblastine

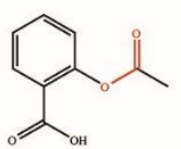

Aspirin

C

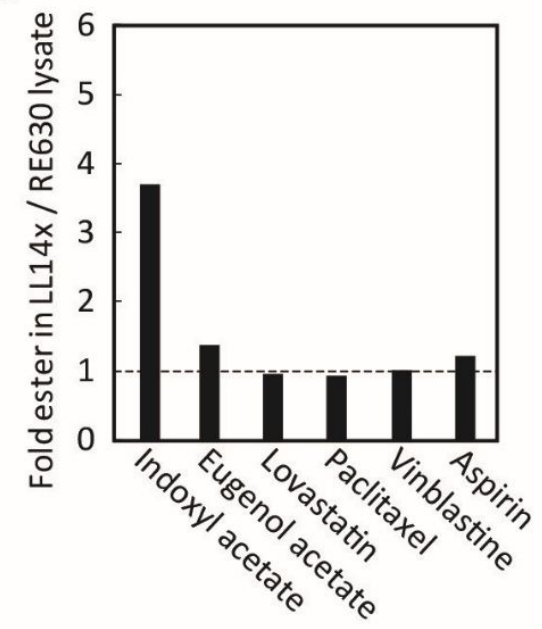


Supporting Figure S3. Ester stability in LL14x hydrolase knockout strain compared to RE630.

(A) Chemical structures of assayed esters. Ester bonds are shown in red and amide bonds are in blue. (B) Triplicate saturated cultures were pelleted and resuspended to OD $=2$ in LB with $400 \mu \mathrm{M}$ of the indicated ester and grown in a 96-well block. After 6 hours for indoxyl acetate and 16 hours for all other esters, cultures were filtered and analyzed by mass spectrometry.

(C) Triplicate cleared lysates were prepared from late-log phase cultures lysed by sonication. To $100 \mu \mathrm{g}$ of protein lysate, $400 \mu \mathrm{M}$ of the indicated ester was added and incubated for 2 hours. Lysates were filtered and analyzed by mass spectrometry. The dashed line indicates no difference between knockout and wild-type samples. 


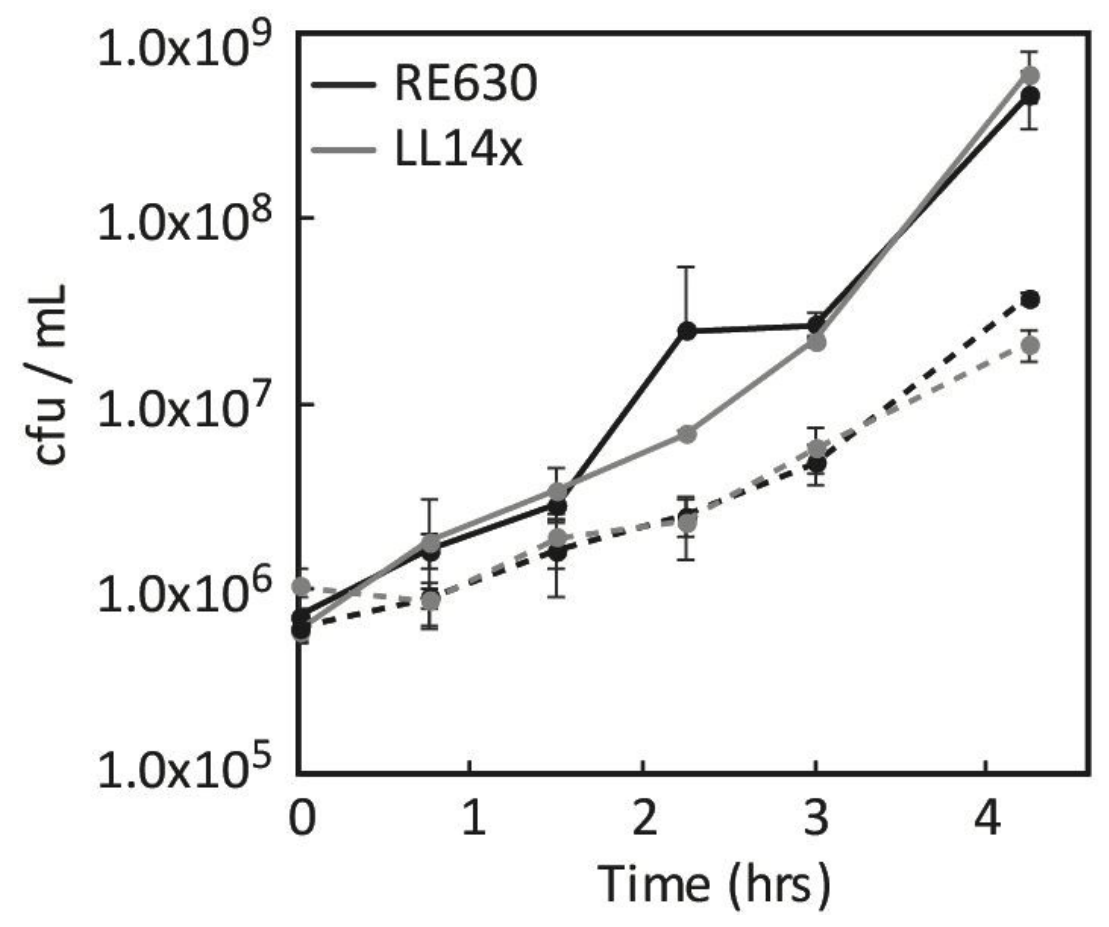

Supporting Figure S4. Indoxyl acetate toxicity is independent of endogenous hydrolysis rate. Saturated RE630 (black) and LL14x (grey) were diluted to OD 0.001 in LB with (dashed) or without (solid) $3 \mathrm{mM}$ indoxyl acetate. At the indicated time points, a serial dilution of cells was spotted on LB + Amp plates. Plates were grown overnight and colonies counted. Error bars represent standard deviation of biological triplicates. 


\section{SUPPORTING METHODS}

Formaldehyde sensitivity. RE630 and the knockout strains were inoculated in $200 \mu \mathrm{L} \mathrm{LB}+$ Amp in a 96-well block and grown overnight. Each culture was diluted 1:220 in $120 \mu \mathrm{L} \mathrm{LB}$ with $(800 \mu \mathrm{M})$ or without formaldehyde in a microtiter plate and grown at $37{ }^{\circ} \mathrm{C}$ in a Sunrise TECAN with high amplitude orbital shaking.

Salicylate sensitivity. RE630 and all knockout strains were inoculated in $200 \mu \mathrm{L}$ LB + Amp in a 96-well block and grown overnight. Each culture was diluted 1:200 in 120 $\mu \mathrm{L} \mathrm{LB}$ with $250 \mu \mathrm{M}$ dipyridyl and 350 $\mu \mathrm{M}$ salicylate in a microtiter plate and grown at $37^{\circ} \mathrm{C}$ in a Sunrise TECAN with high amplitude orbital shaking.

Ester stability in cultures. RE630 and LL14x strains were inoculated in triplicate in $3 \mathrm{~mL}$ of LB + Amp in a 24-well block and grown overnight. Each culture was pelleted and resuspend to OD $=2$ in fresh media. Ester stocks $(100 \mathrm{mM})$ were prepared by dissolving each ester in DMSO. The cultures were split in a 96-well block and each ester $(400 \mu \mathrm{M})$ was added. After 16 hours (6 hours for indoxyl acetate), the cultures were filtered using an AcroPrep Advance 96 Filter Plate. Esters were detected by injecting $5 \mathrm{uL}$ of media on a ZORBAX Eclipse Plus $\mathrm{C}_{18} 2.1 \times 30 \mathrm{~mm} 3.5 \mu \mathrm{m}$ column (Agilent, Santa Clara, CA) kept at $30{ }^{\circ} \mathrm{C}$ connected to a 6520 Accurate-Mass Q-TOF LC-MS (Agilent). Positive mode was used for indoxyl acetate $\left([\mathrm{M}+\mathrm{H}]^{+} 176.06 \mathrm{~m} / \mathrm{z}\right.$; Rt $\left.5.5 \mathrm{~min}\right)$, eugenol acetate $\left([\mathrm{M}+\mathrm{H}]^{+} 207.09 \mathrm{~m} / \mathrm{z}\right.$; Rt $\left.6.4 \mathrm{~min}\right)$, lovastatin $\left([\mathrm{M}+\mathrm{H}]^{+} 405.26 \mathrm{~m} / \mathrm{z}\right.$; Rt $\left.6.5 \mathrm{~min}\right)$, paclitaxel $\left([\mathrm{M}+\mathrm{H}]^{+} 854.33 \mathrm{~m} / \mathrm{z}\right.$; Rt $\left.6.5 \mathrm{~min}\right)$, and vinblastine $\left([\mathrm{M}+\mathrm{H}]^{+} 811.42 \mathrm{~m} / \mathrm{z}\right.$; Rt $\left.5.2 \mathrm{~min}\right)$ with mobile phase A was $0.1 \%$ formic acid in water and mobile phase $B$ was $0.1 \%$ formic acid in acetonitrile. Echinomycin and Ivermectin ([M-H]- $872.51 \mathrm{~m} / \mathrm{z}$; Rt $5 \mathrm{~min}$ ), were run in negative mode using mobile phases containing $0.05 \%$ ammonium hydroxide instead of formic acid. Regardless of mobile phase, the following gradient was used: 0 min 95\% A, $1.5 \mathrm{~min} 95 \% \mathrm{~A}, 7 \mathrm{~min}$ $2 \%$ A, $9 \min 2 \%$ A, $9.5 \min 95 \%$ A, $12 \min 95 \%$ A. Aspirin $\left([\mathrm{M}+\mathrm{H}]^{+} 181.04 \mathrm{~m} / \mathrm{z}\right.$; Rt 1.6-1.7 min) and atropine $\left([\mathrm{M}+\mathrm{H}]^{+} 290.17 \mathrm{~m} / \mathrm{z}\right.$; Rt $\left.1.1 \mathrm{~min}\right)$ used the following gradient in positive mode: $0 \mathrm{~min} 85 \% \mathrm{~A}, 3$ $\min 70 \%$ A, $4 \min 2 \%$ A, $6 \min 2 \%$ A, $6.5 \min 85 \%$ A, 8 min 85\% A. Echinomycin ([M-H]- 1099.42 $\mathrm{m} / \mathrm{z}$; Rt 9.7-9.9 min) was separated using the ZORBAX Eclipse Plus $\mathrm{C}_{18} 4.6 \times 100 \mathrm{~mm} 3.5 \mu \mathrm{m}$ column with the following gradient: $0 \min 95 \% \mathrm{~A}, 3 \mathrm{~min} 95 \% \mathrm{~A}, 14 \mathrm{~min}, 2 \% \mathrm{~A}, 18 \mathrm{~min}, 2 \% \mathrm{~A}, 19 \mathrm{~min}, 95 \% \mathrm{~A}$, $24 \min 95 \% \mathrm{~A}$.

Ester stability in lysate. RE630 and LL14x strains were inoculated in triplicate in $3 \mathrm{~mL}$ of LB + Amp in a 24-well block and grown overnight. Cultures were diluted into $75 \mathrm{~mL}$ of LB + Amp and grown in a 250 $\mathrm{mL}$ baffled shake flask for 5 hours at $37^{\circ} \mathrm{C}$ and $220 \mathrm{rpm}$. Cultures were pelleted, resuspended 1:15 on ice in phosphate buffered saline $\left(137 \mathrm{mM} \mathrm{NaCl}, 10 \mathrm{mM} \mathrm{Na}_{2} \mathrm{HPO}_{4}, 1.8 \mathrm{mM} \mathrm{KH}_{2} \mathrm{PO}_{4}, 2.7 \mathrm{mM} \mathrm{KCl}, \mathrm{pH}=\right.$ 7.4), and sonicated ( $40 \%$ amplitude; $5 \mathrm{~s}$ on, $15 \mathrm{~s}$ off for $60 \mathrm{sec}$ ). Cleared lysates were prepared by pelleting $\left(4{ }^{\circ} \mathrm{C} ; 12,000 \mathrm{xg} ; 20 \mathrm{~min}\right.$ ) and protein concentration was estimated using Bradford reagent (Bio-Rad) with a bovine serum albumin standard curve. For each lysate, $100 \mu \mathrm{g}$ of protein was diluted to $100 \mu \mathrm{L}$ final volume (PBS $+1 \%$ triton $\mathrm{X})$ with ester $(400 \mu \mathrm{M}$ from $100 \mathrm{mM}$ in DMSO). After incubation for two hours at $37^{\circ} \mathrm{C}$, hydrolysis reactions were quenched with $100 \mu \mathrm{L}$ acetonitrile, filtered using the 96 -well filter plate, and run on the QTOF mass spectrometer as described above. 
Indoxyl acetate toxicity. RE630 and LL14x strains were inoculated in $200 \mu \mathrm{L} \mathrm{LB}+$ Amp in a 96-well block and grown overnight. Each culture was diluted to OD 0.001 in $350 \mu \mathrm{L}$ of fresh LB $+\mathrm{Amp}+3 \mathrm{mM}$ indoxyl acetate (prepared using $1 \mathrm{M}$ indoxyl acetate in DMSO). At various times, $20 \mu \mathrm{L}$ of each culture was serially diluted 1:10 and $4 \mu \mathrm{L}$ from each dilution were spotted on a LB + Amp plate. Plates were incubated overnight and colonies in least diluted spot were counted to determine colony forming units (cfu).

\section{SUPPORTING REFERENCES}

1. Gonzalez CF, Proudfoot M, Brown G, Korniyenko Y, Mori H, Savchenko A V., et al. Molecular basis of formaldehyde detoxification: Characterization of two S-formylglutathione hydrolases from Escherichia coli, FrmB and YeiG. J Biol Chem. 2006;281(20):14514-22.

2. Leduc D, Battesti A, Bouveret E. The hotdog thioesterase EntH (YbdB) plays a role in vivo in optimal enterobactin biosynthesis by interacting with the ArCP domain of EntB. J Bacteriol. 2007;189(19):7112-26. 